\title{
An overproduction of astellolides induced by genetic disruption of chromatin-remodeling factors in Aspergillus oryzae
}

\author{
Yasutomo Shinohara $^{1}$, Makoto Kawatani ${ }^{2}$, Yushi Futamura ${ }^{2}$, Hiroyuki Osada ${ }^{2}$ and Yasuji Koyama ${ }^{1}$ \\ The filamentous fungus Aspergillus oryzae is an important industrial mold. Recent genomic analysis indicated that $A$. oryzae has \\ a large number of biosynthetic genes for secondary metabolites (SMs), but many of the SMs they produce have not been \\ identified. For better understanding of SMs production by $A$. oryzae, we screened a gene-disruption library of transcription factors \\ including chromatin-remodeling factors and found two gene disruptions that show similarly altered SM production profiles. One \\ is a homolog of Aspergillus nidulans cclA, a component of the histone 3 lysine 4 (H3K4) methyltransferase complex of proteins \\ associated with Set1 complex, and the other, sppA, is an ortholog of Saccharomyces cerevisiae SPP1, another component of a \\ complex of proteins associated with Set1 complex. The $c c / A$ and $s p p A$ disruptions in $A$. oryzae are deficient in trimethylation \\ of H3K4. Furthermore, one of the SMs that increased in the $c c / A$ disruptant was identified as astellolide $F$ (14-deacetyl \\ astellolide B). These data indicate that both $c c / A$ and $s p p A$ affect production of SMs including astellolides by affecting the \\ methylation status of $\mathrm{H} 3 \mathrm{~K} 4$ in $A$. oryzae.
}

The Journal of Antibiotics (2016) 69, 4-8; doi:10.1038/ja.2015.73; published online 1 July 2015

\section{INTRODUCTION}

Filamentous fungi produce a wide variety of secondary metabolites (SMs). Several SMs with various important properties have been isolated to date. Some such as penicillin (an antibiotic) and lovastatin (a cholesterol-lowering drug) are beneficial for human health, whereas mycotoxins such as aflatoxin and cyclopiazonic acid are detrimental to food and feed safety and human health. ${ }^{1}$ Recent advances in genomesequencing analysis and SM biosynthetic gene cluster prediction using bioinformatics tool such as Secondary Metabolite Unknown Regions Finder ${ }^{2}$ suggested that filamentous fungi have more clusters and SM-producing ability than previously anticipated. ${ }^{3}$ However, the number of SMs that has been isolated to date are fewer than the number predicted from SM gene clusters. One possible reason for this discrepancy is that many SM genes may be inactive (or silent/cryptic/ mutated) under laboratory conditions. ${ }^{4}$ Recently, a variety of methods have been developed to activate silent SM gene clusters in filamentous fungi. ${ }^{5}$ One productive approach is manipulation of epigenetic status by genetically engineering chromatin-remodeling factors or using chemical epigenetic modifiers such as suberoylanilide hydroxamic acid. ${ }^{5-7}$ For example, disruption of the $c c l A$ gene, an ortholog of Saccharomyces cerevisiae BRE2 involved in histone 3 lysine 4 (H3K4) methylation, resulted in activation of two silent SM gene clusters in Aspergillus nidulans $^{8}$ and overproduction of gliotoxin in A. fumigatus. ${ }^{9}$ Overexpression of esaA, an ortholog of $S$. cerevisiae ESAl, led to acetylation of histone 4 lysine 12 and overproduction of four known
SMs (sterigmatocystin, penicillin, terrequinone and orsellinic acid) in A. nidulans..$^{10}$ In A. oryzae, Kawauchi et al. ${ }^{11}$ recently showed that the fungal-specific sirtuin-family histone deacetylase, hstD/Aohst4, affected the production of SMs such as kojic acid and penicillin through the regulation of laeA expression, a global regulator of SM production.

A. oryzae is a fungus widely used for the production of Japanese traditional fermented foods such as soy sauce, miso and sake. ${ }^{12,13}$ A. oryzae is also an important source of industrial enzymes. ${ }^{14}$ Thus, a better understanding of the SMs produced by A. oryzae and their regulation has important implications for obtaining new drug leads and for human health and food safety. The biosynthetic gene clusters for mycotoxins such as aflatoxin, ${ }^{15,16}$ aflatrem $^{17}$ and cyclopiazonic acid ${ }^{18,19}$ have been well characterized, and several genetic causes of failed mycotoxin production in A. oryzae have been identified, whereas the characteristics of other clusters remain unclear.

Our laboratory previously constructed a gene-disruption library of transcription factors in A. oryzae. ${ }^{20}$ As chromatin-remodeling factor disruption strains were included in the library, we investigated alterations of SM production profiles using this library to identify SMs produced by A. oryzae. Here, we report orthologs of S. cerevisiae, $S P P 1$ and BRE2, responsible for the production of different SMs in A. oryzae.

${ }^{1}$ Noda Institute for Scientific Research, Noda, Chiba, Japan and ${ }^{2}$ Chemical Biology Research Group, RIKEN CSRS, Wako, Saitama, Japan Correspondence: Y Shinohara, Noda Institute for Scientific Research, 399 Noda, Noda, Chiba 278-0037, Japan.

E-mail: yshinohara@mail.kikkoman.co.jp

Received 2 April 2015; revised 29 May 2015; accepted 2 June 2015; published online 1 July 2015 


\section{MATERIALS AND METHODS}

\section{General experimental procedures}

Optical rotations were recorded on a SEPA-300 polarimeter (Horiba, Kyoto, Japan). IR spectra were measured with a FT-720 spectrophotometer (Horiba). ${ }^{1} \mathrm{H}-\mathrm{NMR}(500 \mathrm{MHz}),{ }^{13} \mathrm{C}-\mathrm{NMR}(125 \mathrm{MHz}), \mathrm{COSY}$, HSQC, HMBC and NOESY spectra were recorded in DMSO- $d_{6}$ at room temperature with a Bruker AVANCE 500 spectrometer (Bruker, Billerica, MA, USA). Highresolution ESI mass spectrum (HRESI-MS) data were measured using a QSTAR Elite (Applied Biosystems/MDS SCIEX, Foster City, CA, USA), with a mixture of CsI (m/z 132.9054) and sex pheromone inhibitor iPD1 $(\mathrm{m} / \mathrm{z}$ 829.5398) (Applied Biosystems/MDS SCIEX) as the calibration standard.

\section{Fungal strains and culture media}

A gene-disruption library of transcription factors and chromatin-remodeling factors in A. oryzae RIB40 was previously constructed in our laboratory. ${ }^{20}$ This library was used for preliminary metabolite profile screening by HPLC with ESI time-of-flight MS (LC/ESI-TOF MS) (see below). RkuptrP2-1 $\Delta \mathrm{AF} / \mathrm{P}$ (RkuptrP2-1 $\Delta \mathrm{AF}$ host strain with an introduced $p y r G$ gene) was used as a control strain. Czapek yeast (autolysate) extract agar medium (3\% sucrose, $0.5 \%$ yeast extract, $0.3 \% \quad \mathrm{NaNO}_{3}, \quad 0.1 \% \quad \mathrm{~K}_{2} \mathrm{HPO}_{4}, \quad 0.05 \% \mathrm{KCl}, \quad 0.05 \%$ $\mathrm{MgSO}_{4} \cdot 7 \mathrm{H}_{2} \mathrm{O}, 0.001 \% \mathrm{FeSO}_{4} \cdot 7 \mathrm{H}_{2} \mathrm{O}$ and $2 \%$ agar, $\mathrm{pH}$ 6.0) was used as the culture medium for metabolite production. Polypeptone-dextrin medium ( $2 \%$ dextrin, $1 \%$ polypeptone, $0.5 \% \mathrm{KH}_{2} \mathrm{PO}_{4}, 0.1 \% \mathrm{NaNO}_{3}, 0.1 \%$ casamino acids and $0.05 \% \mathrm{MgSO}_{4} \cdot 7 \mathrm{H}_{2} \mathrm{O}, \mathrm{pH} 6.0$ ) was used for preparation of whole-cell extracts.

\section{Metabolite extraction and analysis}

A. oryzae disruptants and the control strain were inoculated onto Czapek yeast extract agar plate and incubated for 7 days at $30^{\circ} \mathrm{C}$. The agar plate with the mycelium was drilled with a cork bowler (diameter, $6 \mathrm{~mm}$ ) and taken as a plug. Ten plugs were placed in a $4 \mathrm{ml}$ glass vial, and $2 \mathrm{ml}$ ethyl acetate was added. The plugs were ultrasonicated for $15 \mathrm{~min}$ and incubated for $15 \mathrm{~min}$ at room temperature. The extracts were transferred into a $2 \mathrm{ml}$ polypropylene tube and centrifuged at $13500 \mathrm{rpm}$ for $10 \mathrm{~min}$. The supernatants $(1.6 \mathrm{ml})$ were dried in a vacuum centrifuge at room temperature and dissolved in $160 \mu$ acetonitrile, and $5 \mu \mathrm{l}$ was injected for LC/ESI-TOF MS analysis. LC/ESI-TOF MS analysis was carried out under the conditions described previously. ${ }^{18}$

\section{Construction of the $c c l A$ and $s p p A$ disruption strains}

Based on the screening results of 111 gene-disruption strains of transcription factors, we selected and further analyzed the following two disruptants: A. nidulans cclA homolog (AO090124000076) and S. cerevisiae SPP1 ortholog

Table 1 PCR primers used in this study

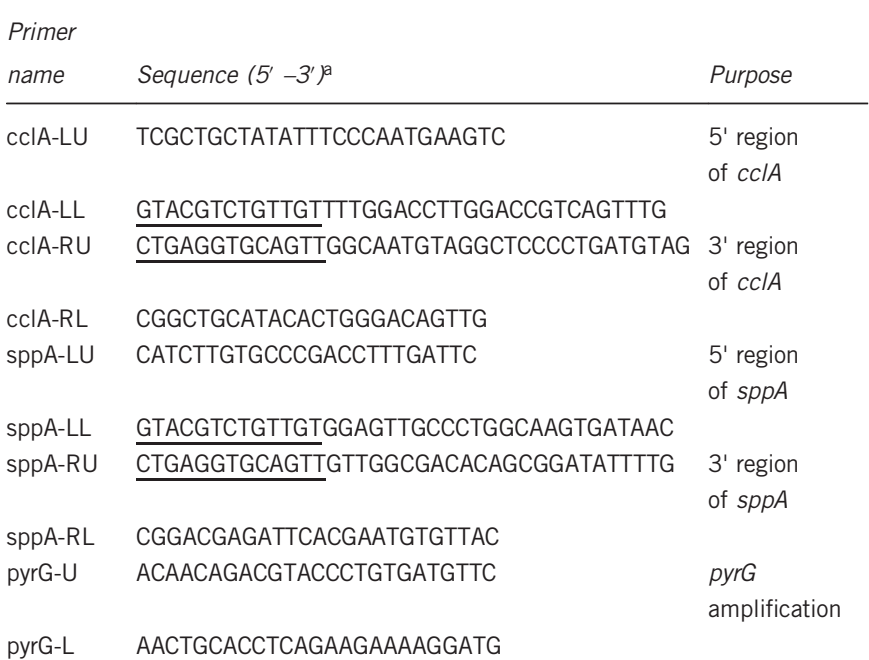

aUnderlined sequences represent the additional nucleotides for fusion PCR.
(AO090003001570). The gene-disruption cassettes were constructed by fusion PCR. In brief, cassettes were constructed with the following three PCR fragments: the $5^{\prime}$-region of the targeted gene was amplified using X-LU and $\mathrm{X}$-LL primers, the $3^{\prime}$-region was amplified using X-RU and X-RL primers, and an auxotrophic marker $p y r G$ was amplified using pyrG-U and pyrG-L primers. Each fragment was amplified from the genomic DNA of A. oryzae RIB 40. Three amplified fragments were mixed and subjected to PCR with primers $\mathrm{X}-\mathrm{LU}$ and X-RL, where ' $\mathrm{X}$ ' represents the name of each target gene. The resulting PCR products were purified and used as a transformation vector. Transformation was performed using the protoplast-polyethylene glycol method described previously. ${ }^{18}$ The primers used for PCR are summarized in Table 1.

\section{Western blot analysis}

Western blot analysis was performed as described previously. ${ }^{21}$ Whole-cell extracts were prepared from mycelium grown for $24 \mathrm{~h}$ in polypeptone-dextrin medium. H3K4 methylation levels were analyzed with anti-H3K4 monomethylation (Active Motif, Carlsbad, CA, USA), anti-H3K4 dimethylation (Merck Millipore, Billerica, MA, USA), anti-H3K4 trimethylation (Active Motif) and anti-histone 3 (Active Motif). Immunoreactive bands were detected using a horseradish peroxidase-conjugated secondary antibody (Thermo Scientific, Rockford, IL, USA) followed by SuperSignal West Pico Substrate (Thermo Scientific)

\section{Isolation of 14-deacetyl astellolide $A$ and $B$}

The $c c l$ disruption strain was cultured at $30^{\circ} \mathrm{C}$ for 7 days on 11 Czapek yeast extract agar medium (40 plates). The fungal culture was chopped into small pieces and extracted with two volumes of ethyl acetate twice. The combined extracts were dried by a rotary evaporator, redissolved in $40 \mathrm{ml} 50 \%$ acetonitrile and centrifuged for $30 \mathrm{~min}$. The resulting supernatant was filtered through a $0.45-\mu \mathrm{m}$ filter (Merck Millipore). The filtrates were purified by reverse-phase HPLC. The HPLC conditions were as follows: column, COSMOSIL 5C18-AR-II $(20 \times 250 \mathrm{~mm})$ (Nacalai Tesque, Kyoto, Japan); flow rate, $5 \mathrm{ml} \mathrm{min}^{-1}$; solvent $\mathrm{A}, 0.1 \%$ formic acid in water; solvent $\mathrm{B}, 0.1 \%$ formic acid in acetonitrile; gradient profile, $0-10 \mathrm{~min}, 0-40 \% \mathrm{~B} ; 10-35 \mathrm{~min}, 40-80 \%$ B, 35-36 min, $80-100 \%$ B; $36-45 \mathrm{~min}, 100 \%$ B; $45-50 \mathrm{~min}, 100-0 \%$ B. UV detection was performed at $260 \mathrm{~nm}$. 14-deacetyl astellolide A (14-DAA) (1) $(9.1 \mathrm{mg})$ and 14-deacetyl astellolide B (14-DAB) (2) $(5.6 \mathrm{mg})$ were eluted at 42.0 and $37.2 \mathrm{~min}$, respectively.

14-DAA (1): colorless solid; $[\alpha]_{589}^{24}-17^{\circ}(c=0.13, \mathrm{MeOH})$; IR (attenuated total reflectance) $3417,2935,2870,1736,1714,1381,1265,1230,1176,1093$, 1065, $1020 \mathrm{~cm}^{-1}$; molecular formula: $\mathrm{C}_{24} \mathrm{H}_{28} \mathrm{O}_{7}$; HRESI-MS $(\mathrm{m} / \mathrm{z})$ : $[\mathrm{M}+\mathrm{H}]$ ${ }^{+}=429.1907$ (calculated for $\mathrm{C}_{24} \mathrm{H}_{29} \mathrm{O}_{7}, 429.1913$ ). The ${ }^{1} \mathrm{H}$ and ${ }^{13} \mathrm{C}$ NMR spectral data are presented in Supplementary Table S1.

14-DAB (2): colorless solid; $[\alpha]_{589}^{25}-9^{\circ}(c=0.11, \mathrm{MeOH})$; IR (attenuated total reflectance) $3373,2918,2850,1734,1706,1601,1591,1446,1381,1265$, $1234,1163,1088,1066,1020 \mathrm{~cm}^{-1}$; molecular formula: $\mathrm{C}_{24} \mathrm{H}_{28} \mathrm{O}_{8}$; HRESI-MS $(\mathrm{m} / \mathrm{z}):[\mathrm{M}+\mathrm{H}]^{+}=445.1863$ (calculated for $\mathrm{C}_{24} \mathrm{H}_{29} \mathrm{O}_{8}, 445.1862$ ). The ${ }^{1} \mathrm{H}$ and ${ }^{13} \mathrm{C}$ NMR spectral data are presented in Supplementary Table S1.

\section{In vitro antiproliferative activity}

In vitro antiproliferative activity was performed by using the human cervix cancer cell line (HeLa), human acute promyelocytic leukemia cell line (HL-60), mouse temperature-sensitive cdc2 mutant breast cancer cell line (tsFT210) and rat temperature-sensitive $v$-src mutant fibroblast cell line $\left(s r c^{\text {ts }}\right.$ NRK) as described previously. ${ }^{22-24}$ In brief, HeLa cells $\left(4 \times 10^{3}\right.$ cells per well $200 \mathrm{\mu l}^{-1}$ ) were seeded on a 96-well microtiter plate (IWAKI, Asahi Glass Co., Ltd, Tokyo, Japan) and incubated overnight. HL-60 cells $\left(1.5 \times 10^{4}\right.$ cells per well $\left.200 \mu \mathrm{l}^{-1}\right)$, tsFT210 cells $\left(1.6 \times 10^{4}\right.$ cells per well $\left.200 \mu \mathrm{l}^{-1}\right)$ and $s r c^{\text {ts }}-\mathrm{NRK}$ cells $\left(1 \times 10^{4}\right.$ cells per well $\left.200 \mathrm{\mu l}^{-1}\right)$ were seeded on 96-well microtiter plates and incubated for $3 \mathrm{~h}$. Serially diluted solutions of the test compounds were added to $0.5 \%(\mathrm{v} / \mathrm{v})$ final concentration. After $48 \mathrm{~h}$ treatment, cell growth was measured using Cell Count Reagent SF (Nacalai Tesque) according to the manufacturer's instructions. In brief, $20 \mu$ WST- 8 solution was added to each well, and HeLa cells and HL-60 cells were incubated at $37^{\circ} \mathrm{C}$ for $1 \mathrm{~h}$, whereas tsFT210 cells and $s r c^{\text {ts }}$-NRK cells were incubated at $32^{\circ} \mathrm{C}$ for $1 \mathrm{~h}$. Cell growth 
a
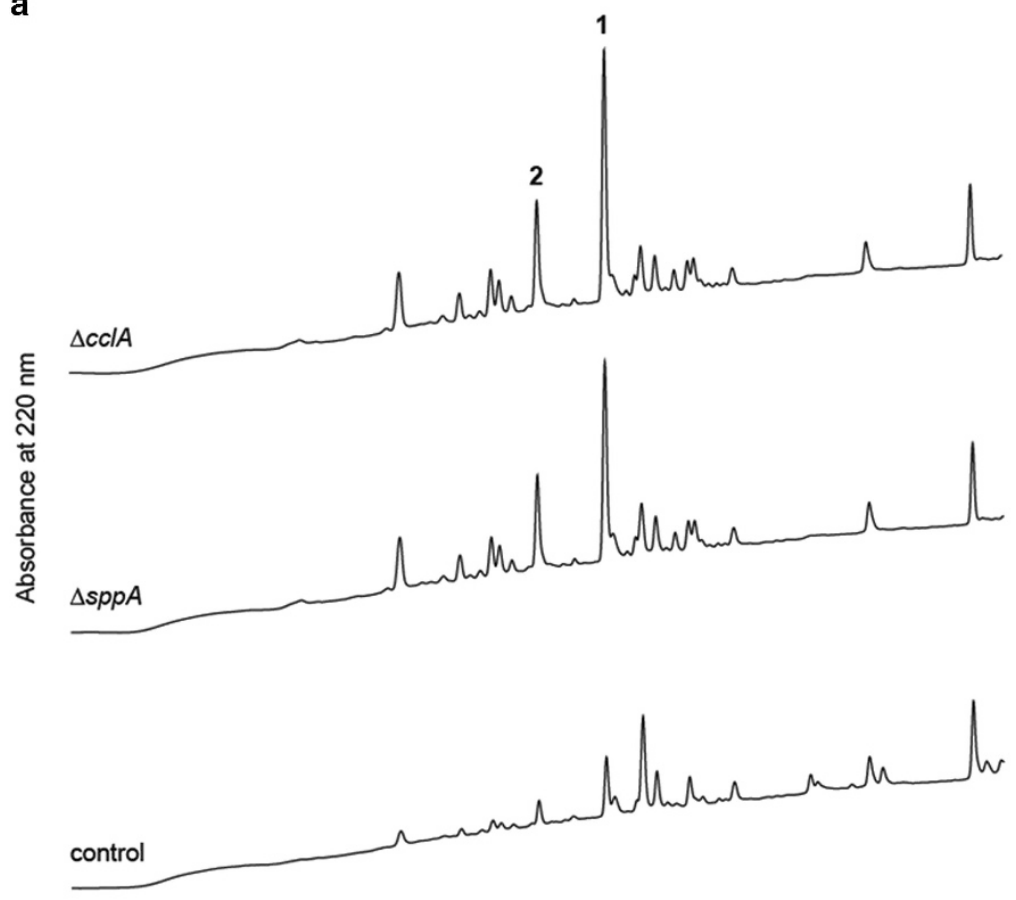

10.011 .012 .013 .014 .015 .016 .017 .018 .019 .020 .021 .022 .023 .024 .025 .026 .027 .028 .029 .030 .0

Time, $\min$ b

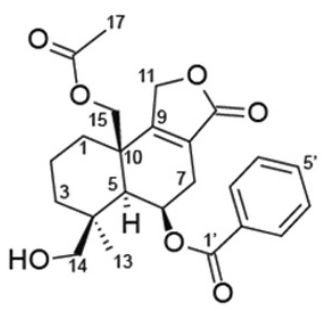

14-deacetyl astellolide $A(1)$<smiles>CC(=O)OC[C@]12CCC[C@@](C)(CO)C1C(OC(=O)c1ccc(O)cc1)CC1=C2COC1=O</smiles>

14-deacetyl astellolide B (2)

Figure $1 \mathrm{HPLC}$ profiles of the culture extracts of the $\operatorname{ccl}(\Delta c c / A)$ and $\operatorname{spp} A(\Delta \operatorname{spp} A)$ disruption strains. (a) UV trace at $220 \mathrm{~nm}$. (b) Chemical structures of 14-deacetyl astellolide A (1) and B (2).

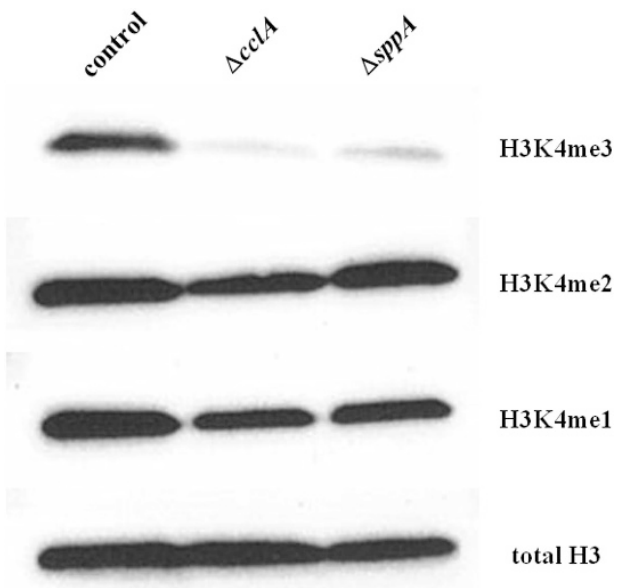

Figure 2 The level of mono-, di- and trimethylated H3K4 in the $c c / A(\triangle c c / A)$ and $\operatorname{spp} A(\triangle \operatorname{spp} A)$ disruption strains.

was measured from the absorbance at $450 \mathrm{~nm}$ on a microplate reader (Perkin Elmer, Norwalk, CT, USA).

\section{RESULTS}

Disruption of complex of proteins associated with Set1 component genes causes enhanced production of several SMs

The screening results revealed similar alterations in the HPLC profiles of two disruption strains compared with that of the control strain (Figure 1a). A BLAST search using the amino-acid sequence of each disrupted gene revealed that one (AO090124000076) shared 75.8\%
Table 2 Antiproliferative activity of 14-deacetyl astellolide A and B

Antiproliferative activity $\left(I_{50}, \mu \mathrm{M}\right)$

\begin{tabular}{lrr} 
Cell lines & $14-D A A$ & $14-D A B$ \\
\hline HeLa & $>30$ & 5.6 \\
HL-60 & $>30$ & 1.8 \\
tsFT210 & $>30$ & 16.1 \\
srcts-NRK & $>30$ & 17.4 \\
\hline
\end{tabular}

overall sequence identity with $A$. nidulans $\mathrm{CclA}$, so we identified this gene as A. oryzae cclA. The other (AO090003001750) showed a high similarity at the $\mathrm{C}$ terminus with $S$. cerevisiae Spplp. The Pfam database search further revealed a conserved plant homeodomain finger, which is also present in $S$. cerevisiae Spplp. Thus, we considered this gene product an ortholog of S. cerevisiae SPP1 and named it $\operatorname{spp} A$.

S. cerevisiae SPP1 and BRE2, the A. nidulans cclA ortholog in $S$. cerevisiae, are well-studied components of the complex of proteins associated with Set1 complex, which regulates $\mathrm{H} 3 \mathrm{~K} 4 \mathrm{di}-$ and trimethylation. ${ }^{21}$ Thus, we examined the H3K4 methylation status in A. oryzae cclA and sppA disruption strains by western blotting. We found that the level of $\mathrm{H} 3 \mathrm{~K} 4$ trimethylation was markedly diminished, whereas those of $\mathrm{H} 3 \mathrm{~K} 4$ di- and monomethylation were unaltered in either the $c c l A$ or the $\operatorname{sppA}$ disruption strain (Figure 2).

\section{Identification of 14-deacetyl astellolide A and B}

To identify the compounds that were overproduced by $c c l A$ disruption, compounds corresponding to peaks 1 and 2 ( 1 and 2 ) were 
isolated from the large-scale culture of the $c c l A$ disruption strain. The contents of compounds $\mathbf{1}$ and $\mathbf{2}$ in the culture extract of the $c c l A$ disruption strain were increased 3.7- and 4.6-fold, respectively, as compared with those in the control strain. The structural characteristics of these compounds were determined by NMR and MS. Compound 1 was identified as a C14-deacetylated derivative of parasiticolide A/astellolide A (14-DAA), which was recently isolated from A. oryzae RIB40, by comparison of its NMR and MS spectra with those previously reported (Figure $1 \mathrm{~b}) .{ }^{25}$ Based on the spectral analysis of NMR and MS, compound 2 was identified as a C14-deacetylated derivative of astellolide $\mathrm{B}$ (14-DAB). This compound is identical to astellolide $\mathrm{F}$, which was isolated most recently by Ren et al. ${ }^{26}$ in independent experiments from A. oryzae QXPC-4. The detail structure analysis of 14-DAB (astellolide F) is described in Supplementary Information.

\section{Only 14-DAB has an antiproliferative activity}

The antiproliferative effects of 14-DAA and 14-DAB on four cancer cell lines (HeLa, HL-60, tsFT210 and $s r c^{\text {ts }}-\mathrm{NRK}$ ) were evaluated from viable cell counts using a WST-8 assay. The antiproliferative activity was measured for different concentrations of 14-DAA and 14 -DAB $(0.1,0.3,1,3,10$ and $30 \mu \mathrm{M})$ after $48 \mathrm{~h}$ of treatment in each cell line. 14-DAB showed antiproliferative activity on all four cell lines, whereas 14-DAA did not show any activity at the concentrations studied. The $\mathrm{IC}_{50}$ values of $14-\mathrm{DAB}$ were summarized in Table 2.

\section{DISCUSSION}

In this study, we revealed that disruption of $c c l A$ altered the SM production profiles in A. oryzae. This result is consistent with previous reports that $c c l A$ disruption caused overproduction of some SMs in A. nidulans and A. fumigatus. ${ }^{8,9}$ Furthermore, we found that disruption of $\operatorname{sp} P A$ in $A$. oryzae showed a similar SM profile to that of the $c c l A$ disruption strain. In S. cerevisiae, BRE2 and SPP1, which are orthologs of $c c l A$ and $\operatorname{spp} A$, respectively, are well-known components of the complex of proteins associated with Set1 complex, ${ }^{21}$ a $\mathrm{H} 3 \mathrm{~K} 4$ methyltransferase. A previous study reported that disruption of these two genes resulted in decreased $\mathrm{H} 3 \mathrm{~K} 4$ trimethylation in S. cerevisiae. ${ }^{21}$ Although tri- and dimethylation of $\mathrm{H} 3 \mathrm{~K} 4$ are generally associated with active transcription, they are required for gene silencing in some cases such as mating type loci and sub-telomeric loci. ${ }^{21,27}$ Our finding that the overproduction of SMs upon $c c l A$ disruption in $A$. oryzae together with previous reports in A. nidulans $^{8}$ and $A$. fumigates $^{9}$ suggests that $\mathrm{H} 3 \mathrm{~K} 4$ methylation is commonly associated with SM gene cluster silencing in Aspergillus spp. Further analysis is needed to elucidate the regulation mechanism of SMs production via chromain remodeling.

Two compounds that were overproduced by $c c l A$ disruption in A. oryzae RIB40 were isolated and identified as 14-DAA and 14-DAB; the latter is identical to astellolide $\mathrm{F}$, recently isolated from liquid culture of A. oryzae QXPC- $4 .{ }^{26}$ Parasiticolide A/astellolide A, which is acetylated at C14 of 14-DAA, was originally isolated from A. parasiticus $^{28}$ and later from a mutant strain of A. variecolor. ${ }^{29}$ In A. variecolor, astellolide $\mathrm{B}$, which is para-hydroxylated at the benzoate moiety of astellolide A, was also isolated. ${ }^{29}$ A previous study reported that $A$. oryzae RIB40 might lose the ability to synthesis of astellolide A, whereas it was able to produce 14 -DAA. ${ }^{25}$ But, most recently nine astellolide analogs including astellolide $\mathrm{A}$ and $\mathrm{B}$ from A. oryzae QXPC-4 were reported. ${ }^{26}$ We attempted to confirm whether these two acetylated compounds of 14-DAA and $14-\mathrm{DAB}$ could be detected in the culture extract of the $c c l A$ disruption strain in A. oryzae RIB40. Using liquid chromatography-MS analysis, the peak at $\mathrm{m} / \mathrm{z} 493$ corresponding to the sodium adduct of astellolide A was slightly detectable in the culture extract of the cclA disruption strain but not in that of control strain; however, the peak intensity was too weak to identify by MS/MS analysis. On the other hand, the peak for astellolide B $(\mathrm{m} / \mathrm{z} 509)$ was undetectable. In addition, Rank et al. ${ }^{25}$ reported that another astellolide analog, which is deacetylated at C15 of 14-DAA, was also isolated from the extract of $A$. oryzae RIB40. It is conceivable that this analog is an intermediate of 14-DAA synthesis. Therefore, we also evaluated the presence of dideacetyl derivative and its hydroxylated derivative (hereafter referred to as dideacetyl astellolide A and B) by MS/MS analysis and detected them in the culture extract of the $c c l A$ disruption strain in A. oryzae RIB40. Furthermore, the peak intensities corresponding to sodium adduct of dideacetyl astellolide A $(\mathrm{m} / z$ 409) and $\mathrm{B}(\mathrm{m} / \mathrm{z} 425)$ in the $c c l A$ disruption strain were much higher than that in the control strain. $c c l A$ disruption led to the overproduction of 14-DAA and 14-DAB and their biosynthetic intermediate at readily detectable levels but not astellolide A or B, suggesting that 14-DAA and 14-DAB may be end-product of astellolide biosynthetic pathway in A. oryzae RIB40. Further analysis is needed to clarify whether $A$. oryzae RIB40 has the ability to produce di-acetylated derivative of astellolide or end-product of astellolide biosynthesis in A. oryzae varies with the type of strain. In addition, in this study we conducted that astellolides were identified as one of the major products, which were overproduced by the $c c l A$ disruption, whereas there are some other SMs that appear to increase or decrease by the $c c l A$ disruption. For example, the peak at $27.8 \mathrm{~min}(\mathrm{~m} / \mathrm{z} 442)$ was decreased, and the peak at 23.1 and $23.5 \mathrm{~min}$ (both $\mathrm{m} / \mathrm{z}$ 647) were increased in the $c c l A$ and sppA disruptants (see Figure 1a). These peaks at $\mathrm{m} / \mathrm{z} 442$ and $\mathrm{m} / \mathrm{z} 647$ were corresponding to the sodium adducts of 13-desoxypaxilline and ditryptoleucines, respectively, which were recently isolated from the culture extract of $A$. oryzae RIB40. ${ }^{25}$ This result suggested that 13-desoxypaxilline and ditryptoleucines productions were also regulated by $c c l A$; however, further experiments are needed to verify this assumption.

Because the sesquiterpene lactone, which forms the basic skeleton of a astellolide, has a variety of biological activities including tumor suppression, ${ }^{30}$ we next examined the antiproliferative activity of 14-DAA and 14-DAB against four cancer cell lines. Interestingly, only $14-\mathrm{DAB}$ showed activity against all cell lines tested in this study. But this result is contrary to previous report that astellolide $\mathrm{F}(=14-\mathrm{DAB})$ did not have antiproliferative activity against several cell line including HeLa even up to $20 \mu \mathrm{m}{ }^{26}$ One possibility for this difference is that the different methods and strain of cell line were used for viable cell count. The detail analysis regarding its cytotoxic effects and mechanism of action is currently under investigation.

Although, astellolide A was isolated several decades ago, genes responsible for its biosynthesis remain unknown. Thus, further studies are in progress to elucidate the biosynthetic gene cluster of astellolides in A. oryzae using these overproducing mutants and genomic information.

\section{ACKNOWLEDGEMENTS}

We wish to thank H Aono (RIKEN) for her help with the experiments. We also thank T Nogawa (RIKEN) and A Okano (RIKEN) for IR spectra and optical rotation measurements. 
1 Sanchez, J. F., Somoza, A. D., Keller, N. P. \& Wang, C. C. Advances in Aspergillus secondary metabolite research in the post-genomic era. Nat. Prod. Rep. 29, 351-371 (2012).

2 Khaldi, N. et al. SMURF: Genomic mapping of fungal secondary metabolite clusters. Fungal Genet. Biol. 47, 736-741 (2010).

3 Medema, M. H. et al. antiSMASH: rapid identification, annotation, and analysis of secondary metabolite biosynthesis gene clusters in bacterial and fungal genome sequences. Nucleic Acids Res. 39, W339-W346 (2011).

4 Palmer, J. M. \& Keller, N. P. Secondary metabolism in fungi: does chromosomal location matter? Curr. Opin. Microbiol. 13, 431-436 (2010).

5 Brakhage, A. A. \& Schroeckh, V. Fungal secondary metabolites - strategies to activate silent gene clusters. Fungal Genet. Biol. 48, 15-22 (2011).

6 Strauss, J. \& Reyes-Dominguez, Y. Regulation of secondary metabolism by chromatin structure and epigenetic codes. Fungal Genet. Biol. 48, 62-69 (2011).

7 Henrikson, J. C., Hoover, A. R., Joyner, P. M. \& Cichewicz, R. H. A chemical epigenetics approach for engineering the in situ biosynthesis of a cryptic natural product from Aspergillus niger. Org. Biomol. Chem. 7, 435-438 (2009).

8 Bok, J. W. et al. Chromatin-level regulation of biosynthetic gene clusters. Nat. Chem. Biol. 5, 462-464 (2009).

9 Palmer, J. M. et al. Loss of CclA, required for histone 3 lysine 4 methylation, decreases growth but increases secondary metabolite production in Aspergillus fumigates. Peer J. 1, e4 (2013).

10 Soukup, A. A. et al. Overexpression of the Aspergillus nidulans histone 4 acetyltransferase EsaA increases activation of secondary metabolite production. Mol. Microbiol. 86, 314-330 (2012)

11 Kawauchi, M., Nishiura, M. \& Iwashita, K. Fungus-specific sirtuin HstD coordinates secondary metabolism and development through control of LaeA. Eukaryot. Cell 12, 1087-1096 (2013).

12 Machida, M. et al. Genome sequencing and analysis of Aspergillus oryzae. Nature 438, 1157-1161 (2005).

13 Machida, M., Yamada, O. \& Gomi, K. Genomics of Aspergillus oryzae: learning from the history of Koji mold and exploration of its future. DNA Res. 15, 173-183 (2008).

14 Olempska-Beer, Z. S., Merker, R. I., Ditto, M. D. \& DiNovi, M. J. Food-processing enzymes from recombinant microorganisms-a review. Regul. Toxicol. Pharmacol. 45, 144-158 (2006).

15 Watson, A. J., Fuller, L. J., Jeenes, D. J. \& Archer, D. B. Homologs of aflatoxin biosynthesis genes and sequence of afIR in Aspergillus oryzae and Aspergillus sojae. Appl. Environ. Microbiol. 65, 307-310 (1999).
16 Tominaga, M. et al. Molecular analysis of an inactive aflatoxin biosynthesis gene cluster in Aspergillus oryzae RIB strains. Appl. Environ. Microbiol. 72, 484-490 (2006).

17 Nicholson, M. J. et al. Identification of two aflatrem biosynthesis gene loci in Aspergillus flavus and metabolic engineering of Penicillium paxilli to elucidate their function. Appl. Environ. Microbiol. 75, 7469-7481 (2009).

18 Tokuoka, M. et al. Identification of a novel polyketide synthase-nonribosomal peptide synthetase (PKS-NRPS) gene required for the biosynthesis of cyclopiazonic acid in Aspergillus oryzae. Fungal Genet. Biol. 45, 1608-1615 (2008).

19 Kato, N. et al. Genetic safeguard against mycotoxin cyclopiazonic acid production in Aspergillus oryzae. Chembiochem 12, 1376-1382 (2011).

20 Ogawa, M., Kobayashi, T. \& Koyama, Y. ManR, a novel $\mathrm{Zn}(\mathrm{II})_{2} \mathrm{Cys}_{6}$ transcriptional activator, controls the $\beta$-mannan utilization system in Aspergillus oryzae. Fungal Genet. Biol. 49, 987-995 (2012).

21 Mueller, J. E., Canze, M. \& Bryk, M. The requirements for COMPASS and Paf1 in transcriptional silencing and methylation of histone $\mathrm{H} 3$ in Saccharomyces cerevisiae. Genetics 173, 557-567 (2006).

22 Osada, H., Magae, J., Watanabe, C. \& Isono, K. Rapid screening method for inhibitors of protein kinase C. J. Antibiot. 41, 925-931 (1988).

23 Osada, H., Cui, C. B., Onose, R. \& Hanaoka, F. Screening of cell cycle inhibitors from microbial metabolites by a bioassay using a mouse cdc2 mutant cell line, tsFT210. Bioorg. Med. Chem. 5, 193-203 (1997).

24 Osada, H., Koshino, H., Isono, K., Takahashi, H. \& Kawanishi, G. Reveromycin A, a new antibiotic which inhibits the mitogenic activity of epidermal growth factor. J. Antibiot. 44, 259-261 (1991)

25 Rank, C. et al. Comparative chemistry of Aspergillus oryzae (RIB40) and A. flavus (NRRL 3357). Metabolites 2, 39-56 (2012).

26 Ren, R. et al. Drimane sesquiterpenoids from the Aspergillus oryzae QXPC-4. Chem. Biodivers. 12, 371-379 (2015).

27 Krogan, N. J. et al. COMPASS, a histone H3 (Lysine 4) methyltransferase required for telomeric silencing of gene expression. J. Biol. Chem. 277, 10753-10755 (2002).

28 Hamasaki, T. et al. A new metabolite, parasiticolide A, from Aspergillus parasiticus. Agric. Biol. Chem. 39, 749-751 (1975).

29 Gould, R. O., Simpson, T. J. \& Walkinshaw, M. D. Isolation and X-ray crystal structures of astellolides $A$ and $B$, sesquiterpenoid metabolites of Aspergillus variecolor. Tetrahedron Lett. 22, 1047-1050 (1981).

30 Amorim, M. H., Gil-da-Costa, R. M., Lopes, C. \& Bastos, M. M. Sesquiterpene lactones: adverse health effects and toxicity mechanisms. Crit. Rev. Toxicol. 43, 559-579 (2013).

Supplementary Information accompanies the paper on The Journal of Antibiotics website (http://www.nature.com/ja) 